\title{
Implantable in vivo Dosimetric Probe Based on GaN Radioluminescence
}

\author{
Anas Ismail ${ }^{1,4}$, Patrick Pittet ${ }^{2}$, Guo-Neng Lu ${ }^{2}$, Jean-Marc Galvan ${ }^{2}$, Jean-Yves Giraud ${ }^{1}$, and Jacques Balosso ${ }^{1,3}$ \\ ${ }^{1}$ Service de Radiothérapie, Centre Hospitalier Universitaire de Grenoble, BP 217, F-38043 Grenoble Cedex 9, France \\ ${ }^{2}$ Institut des Nanotechnologies de Lyon, INL, CNRS UMR5270; Université de Lyon, Lyon, F-69003, France, \\ Université Lyon 1, Villeurbanne, F-69622, France - INSA de Lyon, Villeurbanne, F-69621 \\ ${ }^{3}$ INSERM U647-ESRF, Grenoble, France \\ ${ }^{4}$ Radioprotection department, Syrian Atomic Energy Commission, P.B. 6091, Damascus-Syria
}

\begin{abstract}
This paper presents an implantable in-vivo dosimetric probe using a small-volume GaN bulk as scintillator. The high light yield of GaN under irradiation in radiotherapy conditions is observed. Heavily-doped n-type GaN is chosen because of enhanced and dominant UV emission. The fabrication process of the probe is described. It is tested using 6 and $18 \mathrm{MV}$ photon beams. Measured results show reproducibility errors of less than $2 \%$ for a delivered dose of $50 \mathrm{cGy}$. The linearity of the measure and its independence of the dose rate are also verified. The probe's output light has both GaN and fiber contributions. The fiber contribution may vary with the beam incidence angle (due to dominant Cerenkov effect), while the GaN does not have such dependence.
\end{abstract}

Keywords - in vivo dosimetry, GaN radioluminescence, fiber optic probe, radiotherapy.

\section{INTRODUCTION}

The current trend in external beam radiotherapy is to implement more sophisticated treatment techniques such as 3D conformational therapy. Such implementation also requires qualified treatment means based on cautious quality insurance procedures. However, erroneous parameters could be difficult to detect and systematical errors could happen leading to radiotherapy accidents. In this context implantable in vivo dosimetry has a critical role of final control of the delivered dose.

For in vivo dosimetry in radiotherapy, most existing technologies are based either on thermally or optically stimulated radioluminescence methods and scintillating materials (OSL, TLD) [1,2], or on the use of semiconductor devices (MOSFET, diodes ...) operated with the radioelectric principle $[3,4]$.

OSL and TLD dosimeters need thermal or optical stimulation schemes to release the trapped charges. Such stimulation processes increase the complexity of instrumentation and do not allow real-time reading of the dose.

Dosimeters based on semiconductor devices (diode or MOSFET transistors) have also disadvantages, such as directional effect, and requirement of electrical connections for real-time measurements.
We present in this paper a new implantable dosimetric probe, which can be connected to a photodetection system via an optical fiber, and can perform real-time dose monitoring. Our presentation includes: key material of the probe and its fabrication, and tests results with discussions.

\section{MATERIAL AND DEVICE FABRICATION}

\section{A. GaN Scintillator}

Our proposed domimetric probe is made of a smallvolume GaN bulk as scintillator. Under irradiation, the GaN crystal produces electron-hole pairs in GaN, which predominantly recombine through band edge radiative transitions to emit UV light. Fig. 1 compares two pictures of a GaN crystal bulk before and under 6-MV photon-beam irradiation from a radiotherapy linear accelerator (Elekta $\mathrm{SLi}$ ). The obtained images taken with a CCD camera show that $\mathrm{GaN}$ is highly radioluminescent.

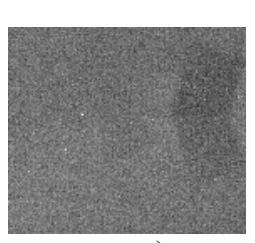

a)

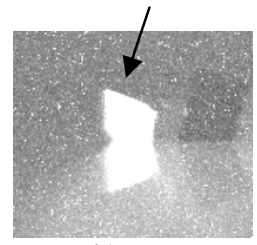

b)
Fig. 1 Pictures of GaN bulk taken with a CCD camera: a) before and b) under irradiation

GaN has a light yield of $\sim 10^{5}$ photons/MeV, significantly higher than scintillating fibers $\left(\sim 8 \times 10^{3}\right.$ photons $/ \mathrm{MeV}$ for $\mathrm{a}$ BCF-12 scintillating fiber from Saint Gobain Crystals) and many conventional scintillating crystals.

Nevertheless, GaN radioluminescence emission can be affected by self absorption within the material. This is why highly doped GaN bulk material is preferred for this dosimetric application: heavy doping shortens radiative recombination time of minority carriers and increases the absorption interband mean free path [5]. We have used 
Si-doped n-type GaN wafers provided by LUMILOG $^{1}$, France. They are strain-free bulk GaN grown by hydridevapor-phase-epitaxy (HVPE), with a shallow donor concentration of $1.5 \times 10^{19} \mathrm{~cm}^{-3}$. Photoluminescence characterization at room temperature of this material shows strong luminescence emission at $3.40 \mathrm{eV}(\sim 365 \mathrm{~nm})$ corresponding to band edge radiative recombinations (see Fig. 2).

In the radiotherapy energy range, Compton effect is the predominant mode of interaction between the beam and the GaN. For x-rays, the mean mass-energy absorption coefficient is an important physical characteristic to consider for dosimetric applications (Fig. 3). GaN is not tissue equivalent, nevertheless for conventional $6 \mathrm{MV}$ and $18 \mathrm{MV}$ radiotherapy photon beams it has a dosimetric response close to that of soft tissues.

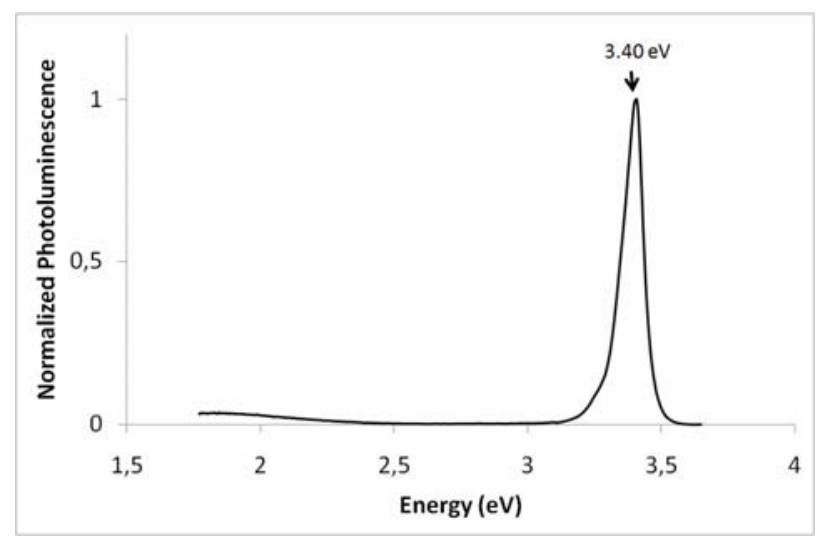

Fig. 2 PL characterization of GaN luminescence at room temperature

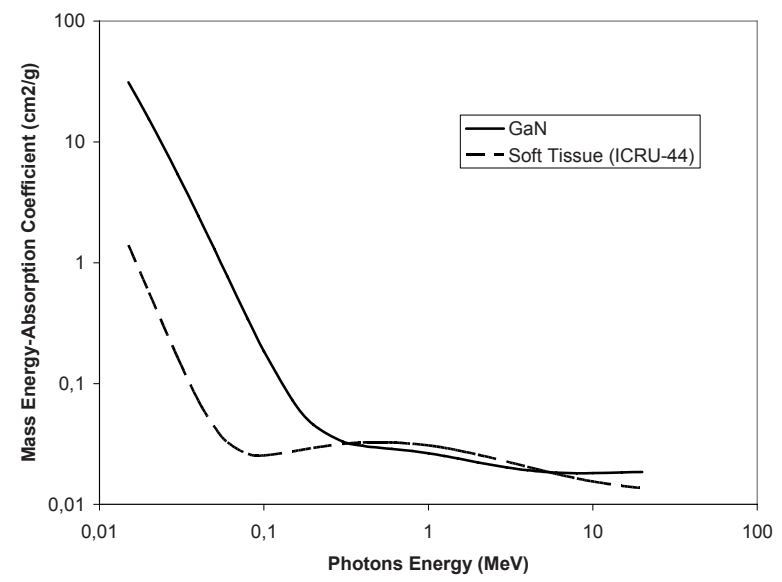

Fig. 3 Mass Energy Absorption Coefficient (NIST data)
$\mathrm{GaN}$ material is robust against irradiation and has been considered to implement detectors for harsh radiation environments [6]. We have applied an industrial gamma sterilization process ( $30 \mathrm{kGy}$ dose from a $\mathrm{Co}^{60}$ source) on a GaN bulk substrate and performed low temperature $(25 \mathrm{~K})$ photoluminescence (PL) characterization of sterilized and non sterilized substrates. No PL spectral changes have been observed for the sterilized substrate.

\section{B. Implantable Dosimetric Probe}

The dosimetric probe is made of a small-volume of GaN bulk (less than $0.01 \mathrm{~mm}^{3}$ ) encapsulated at the distal end of the coupled fiber.

For the coupled optical fiber, we chose a step-index Hard Clad Silica Fiber, HCPM0365T from SEDI Fibres, France. It has a $365 \mu \mathrm{m}$-diameter fused silica core, a $15 \mu \mathrm{m}$-thick polymerhard cladding, and a $730 \mu \mathrm{m}$-external-diameter TEFZEL. It has a large numerical aperture $(=0.37)$ for collecting more efficiently the GaN RL output, and the TEFZEL buffer is biocompatible.

The cavity of the fiber was realized through a four-step process:

- a tip is formed within the polymer cladding of the fiber by an hydrofluidic acid etching of its fused silica core ;

- $\quad$ the formed tip is then mechanically cleaved with a capillary to obtain a cavity ;

- a polished silica rod with some diamond paste is introduced in the cavity to polish the cleaved core surface ;

- $\quad$ the fiber is cleaned in an ultrasound bath and dried.

Small-volume GaN bulks were obtained by dicing a GaN wafer with a precision diamond wire saw (Well Diamond Wire Saws, Inc., USA). The obtained GaN bulks had a square section of about $270 \mu \mathrm{m} \times 270 \mu \mathrm{m}$ and a length equal to the wafer thickness (ranging from $300 \mu \mathrm{m}$ to $1400 \mu \mathrm{m})$.

The encapsulation process mainly consisted in introducing the GaN bulk in the fiber's cavity, and sealing it with polymer material.

This encapsulation configuration preserves the continuity of the optical clad and the TEFZEL buffer (ETFE) for optimal optical and mechanical performances.

A schematic view and a photo of the the fabricated probes are presented on Fig. $4 a$ and $b$.

\footnotetext{
${ }^{1} \mathrm{http}: / /$ www.lumilog.com
} 


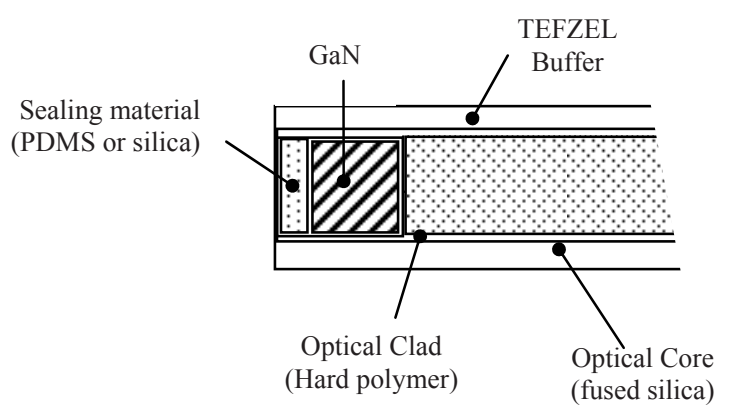

(a)

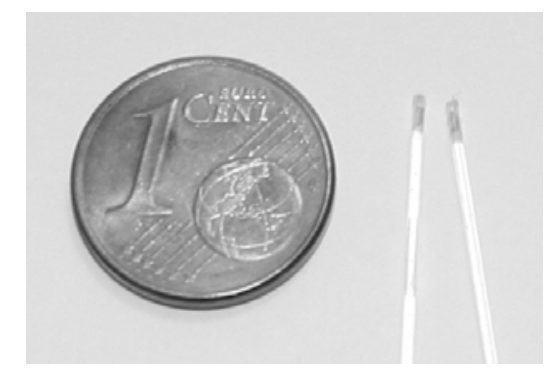

(b)

Fig. 4 a) Schematic view of the distal end of the probe; b) photo of fabricated probes

\section{Probe testing}

Fig. 5 shows the experimental set-up for testing the dosimetric probe. A photodetection module for this purpose was designed. It basically consisted of a $385 \mathrm{~nm} / 10 \mathrm{~nm}$ interferential band-pass filter and a photomultiplier tube (PMT) H6780-20 from Hamamatsu with a transimpedance amplifier. The band-pass filter served to select near-band-edge $\mathrm{RL}$ emission of the GaN signal, so as to minimize wideband background contributions. The amplifier's output was connected to a PC via an acquisition board (Ch-3160, Acquitek) for data recording, monitoring and processing. The dosimetric probe under test was connected via a fiber cable to the photodetection module.

The distal end of the probe was placed into a tissue equivalent material (PMMA) at depths of $1.5 \mathrm{~cm}$ and $3 \mathrm{~cm}$ respectively for $6 \mathrm{MV}$ and $18 \mathrm{MV}$ photon beams.

The dosimetric probe was tested with radiotherapy photon beams from a Varian $600 \mathrm{CD}$ linear accelerator.

\section{A. Reproducibility}

This test consisted of a constant dose of 1.5 Gy delivered fifty times with a dose rate of $300 \mathrm{MU} / \mathrm{min}$ (30s of irradiation). The photodetected signal for the fifty runs was reproducible and remained within an interval of $+/-1.5 \%$.

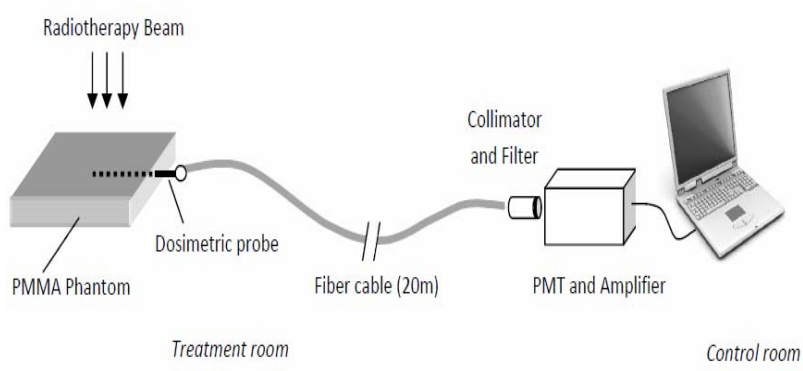

Fig. 5 Experimental set-up for testing the $\mathrm{GaN}$ dosimetric probe

\section{B. Linearity}

The linear response of the probe was tested with a $6 \mathrm{MV}$ photon beam. Both the probe under test and an ionization chamber (as reference) were placed side-by-side between plates of PMMA at $1.5 \mathrm{~cm}$ deep, and around the center of a field of $10 \times 10 \mathrm{~cm}^{2}$. The obtained results from the probe and the ionization chamber are plotted in Fig. 6, which shows a good agreement and an excellent linearity for doses between 0 and $18 \mathrm{~Gy}$.

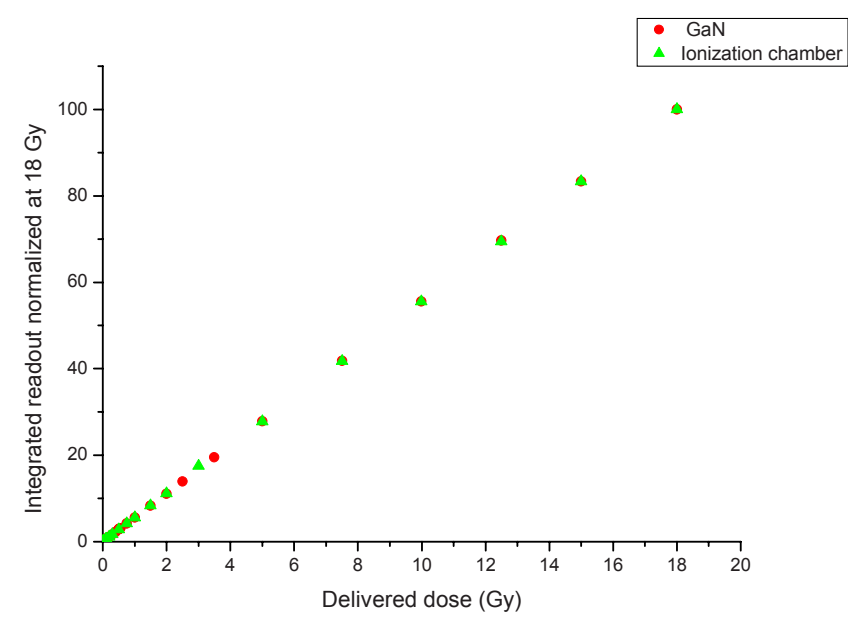

Fig. 6 Linearity of the probe compared with an ionization chamber

\section{Dose Rate Dependence}

Dose rate dependence of the probe was verified from $2 \mathrm{~Gy} / \mathrm{min}$ to $6 \mathrm{~Gy} / \mathrm{min}$. Successively we delivered within one minute a dose of $1 \mathrm{~Gy}$ with dose rate of $1 \mathrm{~Gy} / \mathrm{min}$ and $2 \mathrm{~Gy}$ with a dose rate of $2 \mathrm{~Gy} / \mathrm{min}, . .$. , up to $6 \mathrm{~Gy}$ at a dose rate of 6 Gy/min. Fig. 7 shows the obtained results, exhibiting a perfect linearity as a function of dose rate. 


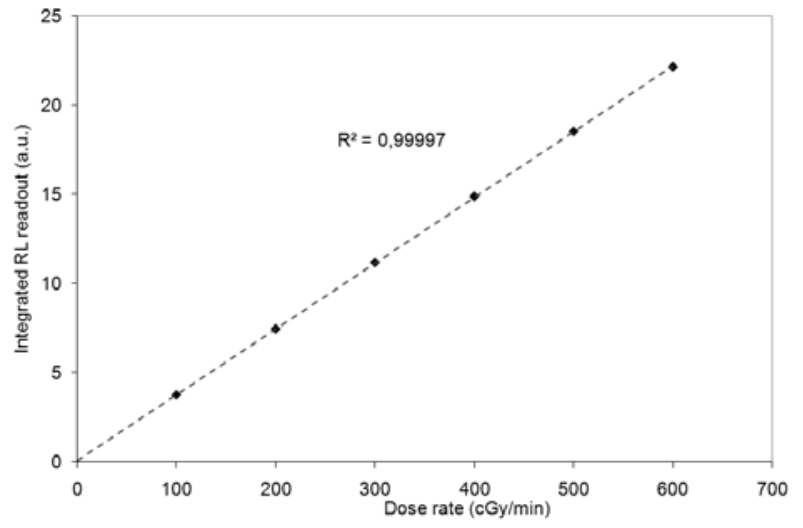

Fig. 7 Dose rate dependence of the probe

\section{GaN/Fiber Contributions}

It should be noted that the probe under test has a segment of the coupled fiber in the radiation field. Therefore the probe's output light has both $\mathrm{GaN}$ and fiber contributions. The fiber contribution consists of Cerenkov radiation, and fluorescence or luminescence emission [7].

To estimate $\mathrm{GaN}$ and fiber contributions, we applied series of measurements with different field sizes. Increasing the field size means to increase the irradiated length of the fiber, or its irradiated volume. Fig. 8 confirms that the fiber contribution increases with the fiber irradiated volume. The increase of fiber contribution with higher radiation energy may be accounted for by the Cerenkov effect, which has a strong energy dependence. From the measured results, it can be estimated that $\mathrm{GaN}$ contribution per volume is, respectively, 186 times that of the fiber for $6 \mathrm{MV}$ photons, and 89 times that of the fiber for $18 \mathrm{MV}$ photons.

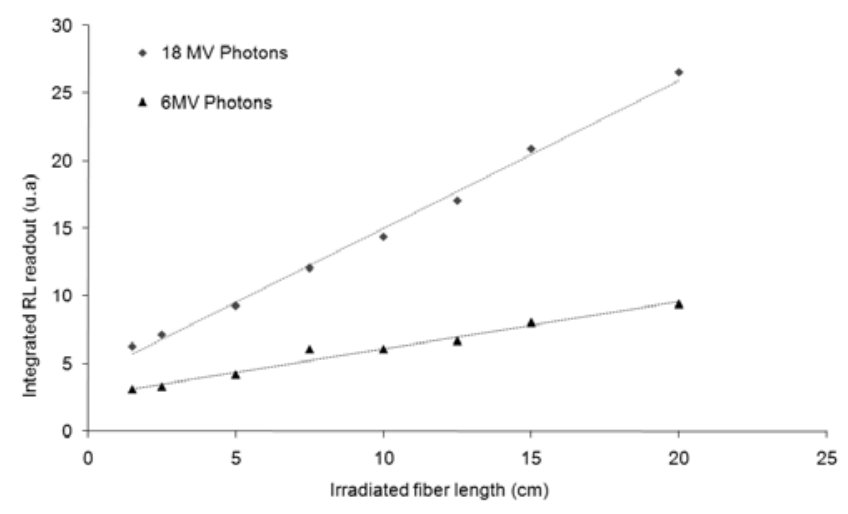

Fig. 8 Probe's output as a function of field size, for $6 \mathrm{MV}$ and 18MV photon beams

\section{E. Angular Dependence}

For testing angular dependence of the probe, it was placed at the isocentre of the field under a constant depth of $1,5 \mathrm{~cm}$ of PMMA plates for each incidence beam angle. Fig. 9 shows measured results using a $18 \mathrm{MV}$ photon beam. It can be seen that increasing the incident beam angle increases the slope of the corresponding curve. This means the increase of fiber contribution, and may be due to dominant Cerenkov effect. At the same time, we can note that these curves cross with the Y-axe at the same point, which means that the GaN contribution is angle-independent.

\section{Conclusions}

We have presented an implantable dosimetric probe using $\mathrm{GaN}$ as scintillator. Its fabrication process used in our preliminary studies has also been described. Testing of the probe using photon beams has shown that it has a linear and reproducible response $(<2 \%$ error), and does not have a dose rate dependence. The probe's output light has both $\mathrm{GaN}$ and fiber contributions. The fiber contribution may vary with incident beam angle, while the GaN contribution is angle-independent. The probe is able to easily resist to gamma ray sterilization process as used for disposable material in medicine.

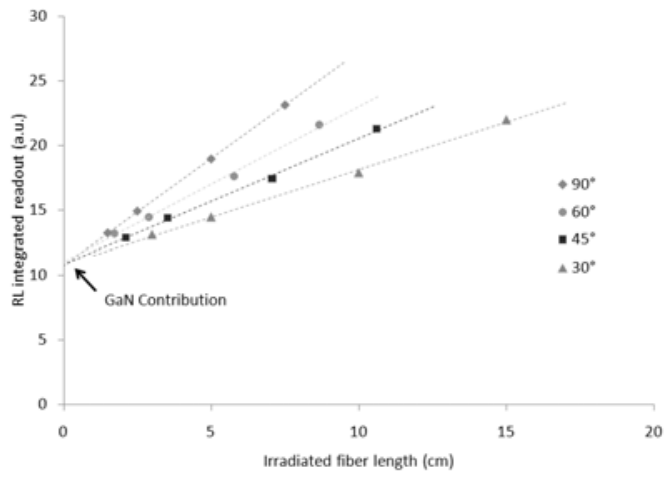

Fig. 9 Probe's output as a function of field size, for a 18MV photon beam, with different incident angle

\section{AcKNowledgment}

"This work is supported by French National Agency (ANR) through Emergence and maturation of projects in Biotechnologies program (Project SECURIDOSE $n^{\circ}$ ANR07-EMPB-028) ". 


\section{REFERENCES}

1. M. C. Aznar, C. E. Andersen, L. B. Jensen, S. A. J. Back, S. Mattsson, F. Kjaer-Kristoffersen, J. Medin, "Real-time optical-fiber luminescence dosimetry for radiotherapy: physical characteristics and applications in photon beams", Phys. Med. \& Biol., 49, pp. 1655-1669, 2004

2. N. A. El-Faramawy, H. Y. Goksu, and W. Panzer, " Thermoluminescence dosimetric properties of a new thin beta detector $(\mathrm{LiF}: \mathrm{Mg}, \mathrm{Cu}, \mathrm{P}$; GR-200F) in comparision with highly sensitive Al2O3:C beta dosimeters," J. Radiol. Prot., 24, 273-282 (2004).

3. Holmes-Siedle, "The space charge dosimeter", Nucl. Inst. Meth., 121, pp. 169-179, 1974.

4. G. P. Beyer, G. G. Mann, J. A. Pursley, E. T. Espenhahn, C. Fraisse, D. J. Godfrey, M. Oldham, T. B. Carrea, N. Bolick, C. W. Scarantino, "An implantable MOSFET dosimeter for the measurement of radiation dose in tissue during cancer therapy", IEEE Sens. J., Vol. 8, No. 2, pp. $38-51,2008$
5. A. Kastalsky, S. Luryi, and B. Spivak, "Semiconductor high-energy radiation scintillation detector", Nuclear Instruments and Methods in Physics Research Section A: Accelerators, Spectrometers, Detectors and Associated Equipment, Volume 565, Issue 2, 15 September 2006, Pages 650-656

6. J. Grant, W. Cunningham, A. Blue, V. O'Shea, J. Vaitkus, E. Gaubas and M. Rahman, "Wide bandgap semiconductor detectors for harsh radiation environments", Nuclear Instruments and Methods in Physics Research Section A: Accelerators, Spectrometers, Detectors and Associated Equipment, Volume 546, Issues 1-2, 1 July 2005, Pages 213-217

7. S. Beddar, "Plastic scintillation dosimetry and its application to radiotherapy", Rad. Meas., 41, pp.S124- S133, 2007

Corresponding author:

Pr Jacques Balosso

Institute: University Hospital of Grenoble

Street: $\quad$ BP 217

City: F-38043 GRENOBLE

Country: France

Email: JBalosso@chu-grenoble.fr 